\title{
Las Naciones Unidas
}

\author{
por el Dr. TRYGVE LYE, \\ Secretario General de las Naciones Unidas
}

El año transcurrido entre el $1^{\circ}$ de julio de 1948 y el 30 de junio de 1949 ha sido, en conjunto, un año de progreso en el camino de la paz mundial.

Cierto es que el mundo ha pasado este año por un buen número de crisis y sobresaltos. Las rivalidades de un conflicto ideológico han sido presentadas como si constituyeran el único problema de nuestro tiempo, miertras las grandes potencias han continuado sus esfuerzos para reforzar sus posiciones respectivas, antes de que la situación tienda a normalizarse mediante la conclusión de los tratados de paz. Aunque relegadas a un segundo plano, las diferencias entre las grandes potencias, los movimientos de indeperdencia nacional y las convulsiones sociales que se han producido en muchas partes del mundo han contribuído inevitablemente a mantener la tensión internacional. Estas condiciones, que har persistido desde que terminó la guerra, continúan causando ansiedad en todos los pueblos del mundo acerca de las perspectivas de la paz murdial y de la capacidad de las Naciones Unidas para impedir una tercera guerra mundial.

A causa de las divergencias entre las grandes potencias, nada so hizo durante el año para proporcionar al Consejo de Seguridad las fuerzas armadas que le permitieran imponer sus decisiones; y se har realizado escasos progresos en la preparación de un acuerdo sobre el contral de la energía atómica, de otros medios de destrucción en masa y de los armamentos de tipo corriente.

\section{PROGRESOS EN EL CAMINO DE LA PAZ MUNDIAL}

Sin embargo ,el temor a la guerra ha disminuído. La superación de la crisis de Berlín contribuyó notablemente a este resultado. El mundo tiene motivos para estar agradecido a las grandes potencias, cuyo sentido po- 
lítico constructivo hizo posible el acuerdo alcarzado por sus representantes en la sede de las Naciones Unidas, después de varios meses de esfuerzos conciliatorios realizados por la Organización. Este acuerdo ha permitido reanudar, con esperanza, las negociaciones sobre Alemania y Austria.

La acción de las Naciones Unidas en otras partes del mundo ha contribuído también a los progresos realizados en el camino de la paz mundial, previniendo o poniendo fin a guerras que afectaríar a 500 millones de personas. Esta acción -en Palestina, en: Cachemira y en Indonesia - no implicó la utilización ce fuerzas militares o policíacas. En verdad el Consejo de Seguridad no dispone de esas fuerzas. La única fuerza utilizada por las Naciones Unidas ha sido su fuerza moral y su poder de persuasión para obtener transacciones razonables y arreglos pacíficos. En cada caso ha habido fracasos de vez en cuando, pero la influencia de las Naciones Unidas, mediante los procedimientos de mediación y de conciliación, se ha: reafirmado siempre y ha prevalecido finalmente.

Es digno de notar que, en los casos mencionados, que afectaban a casi una cuarta parte de la población del mundo, el Consejo de Seguridad ha actuado con éxito considerable, con sujeción a su regla de unanimidad, en el periodo más crítico de tensión entre las grandes potencias. No quiero decir que la aplicación de la regla de unanimidad no haya suscitado dificultades. Sin embargo, se ha hablado tanto de la supuesta parálisis del Consejo de Seguridad y de la consiguiente incapacidad de las Naciones Unidas para impedir la guerra, que creo debe llamarse la atención sobre los resultados obtenidos por el Consejo de Seguridad y en estas y en algunas otras controversias.

En verdad, los conflictos de Palestina, Cachemira e Indonesia son en cierto modo secundarios respecto a los desacuerdos surgidos cor. motivo del arreglo de la situación europea, que casi han monopolizado la atención mundial. Pero estos asuntos, aunque sean exteriores al conflicto europeo, no son ajenos al bienestar de la raza humara o a la paz mundial.

La creación del estado de Israel en Palestina, sin una gucria de grandes proporiones, es uno de los acontecimentos épicos de la historia, que fone fir: no meramente a treinta años, sino a dos mil de acumulados sufrimientos. amarguras y luchas. Tanto para los cristianos como para los judíos y los musulmanes, Palestina es el símbolo de fuerzas históricas respecto a las cuales el actual conflicto ideológico parece un fenómeno transitorio.

Cuando la India y Pakistán aceptaron la proposiciór: de las Naciones Unidas para poner fin a las hostilidades en el Estado de Cachemira. los 400 millones de personas que habitan esos dos grandes paises, recienremente irdependientes, se libraron de la peor de las calamidades: la guerra civil y religiosa.

En Indonesia, las Naciones Unidas han desempeñado un papel decisivo en sus esfuerzos para obtener una solución pacífica del conflicto entre los Países Bajos e Indonesia, y una transición también pacífica hacia la indeperdencia nacional de 70 millones de indonesios. Estos faíses cuentan con más habitantes que la totalidad de Europa, continerte en el que se concentra la atención universal desde la terminación de la guerra. 


\section{PODEROSA INFLUENCIA DE LAS NACIONES UNIDAS EN MATERIA DE CONCILIACION Y DE MEDIACION}

En estos conflictos, como en el suscitado entre las grandes potencias, las Naciores Unidas han demostrado que pueden ejercer poderosa influencia en materia de concriacion y de mediacion. IVlada ulustra mejor esto como una comparación entre el estado de las relaciones de las grandes potencias antes y después del momento en que el contlicto de Berlín tué planteado ante las Naciones Unidas como una amenaza a la paz.

Cuando tres de las grandes potencias plantearon la cuestión de Berlín al Consejo de Seguridad en septiembre último, en una reclamación contra una cuarta potencia, muchas personas temieron que esta crisis pudiera destruir las Naciones Unidas y aun conducir a una nueva guerra mundial.

Nada de esto ocurrió. En cambio, las fuerzas de moderación y de conciliación se pusieron inmediatamente en movimiento. Los miembros no permanentes del Consejo de Seguridad, bajo la dirección de su presidente, procuraron obtener un acuerdo entre las grandes potencias, para salir del punto muerto al cual se había llegado. La Asamblea General aprobó una resolución en la que se hacía un llamamiento a las grandes potencias y se les invitaba a redoblar sus esfuerzos para conciliar sus diferencias, y el presidente de la Asamblea General y el Secretario General pidieron a las grandes potencias, de conformidad con esta resolución, que procuraran una vez más llegar a un acuerdo sobre el caso de Berlín. Nombrado por el presidente del Consejo de Seguridad, un comité de expertos en cuestiones monetarias, integrado por miembros no permanentes, en el cual participó un representante del Secretario General, trató de hallar solución al conflicto por medio de un plan para establecer una moneda única en Berlín.

Ninguno de estos esfuerzos produjo resultados inmediatos, pero sirvieron en gran medida para atenuar la tensión, disminuir el peligro de guerra y ganar tiempo para permitir que otros factores de conciliación entraran en juego. Finalmente, el resultado fué la reanudación, entre las grandes potencias, de las negociaciones sobre los tratados de paz, después de una interrupción total de diecisiete meses, en el curso de los cuales el Consejo de Ministros de Relaciones Exteriores no se había reunido.

Es fundamental que no perdamos ninguna oportunidad de realizar nuevos progresos hacia la paz en los meses venideros, por cualquier desavenencia que pueda surgir en cuanto al papel de las Naciones Unidas en la prevención de la guerra, o ante los desacuerdos entre las potencias "orientales" y "occidentales". Tal desavenencia, juntc con una insistencia excesiva sobre consideraciones inmediatas, en detrimento de otras má permanentes, podría conducir a una serie de actos u omisiones que terminarían por relegar a las Naciones Unidas a desempeñar un papel de segundo orden en los asuntos mundiales.. No me refiero aquí a los pactos o alianzas regionales o bilaterales, cuyos resultados dependen del espíritu con que se conciertan y se cumplen. Estoy pensando, más bien, en una concepción falsa del papel de las Naciones Unidas, concepción que, en cualauier forma que se manifieste, tendrá como resultado su debilitamiento. La falta de cumplimiento de las obligaciones de la Carta o el no recurrir a los órganos de las Naciones Unidas entran en esta categoría: los es- 
fuerzos auténticos para aplicar la Carta y utilizar plenamente los organos de las Naciones Unidas, necesariamente han de fortalecer a la Organización.

Conviene recordar una vez más los principios fundamentales de San Francisco. Allí, los fundadores de las Naciones Unidas convinieron en que sólo una organización universal, fundada en una carta aceptada universalmente, podría. asegurar la paz; y rechazaron la idea de contentarse con menos. Por consiguiente, colocaron los cimientos de las Naciones Unidas de tal modo que reunieran en la Organización a las fuerzas principales que actúan en todas partes del mundo y no sólo en una u otra.

Contrariamente a las ideas erróneas que han surgido más tarde, nunca se pensó en San Francisco que las Naciones Unidas suprimirían a podrían suprimir las diferencias de intereses o de ideología que observamos actualmente er el mundo. No se creyó que las grandes potencias actuarían siempre unánime y fraternalmente. L.o que los fundadores de las Naciones Unidas creían era que la Organización haría posible que las potencias, grandes y pequeñas, ventilaran sus diferercias dentro de límites pacíficos, y que esto no podría lograrse sin las Naciones Unidas. En definitiva, rechazaban la idea de un conflicto insoluble que sólo pudiera resolverse en el campo de batalla; y proclamaror, por el contrario, el principio de que todos los conflictos, por importantes que sean, deben y pueden resolverse por medios pacíficos.

Los resultados obtenidos por las Naciones Unidas en los últimos doce meses han confirmado abundantemente la razón de la doctrina de Sar. Francisco, en vez de hacer surgir dudas sobre ella.

Las Naciones Unidas no han estado en condiciones de resolver las diferencias entre las grandes potencias, pero el conflicto se ha mantenido dentro de límites pacíficos, y se ha preparado el camino para llegar a un arrreglo.

\section{INICIATIVAS DE LAS NACIONES UNIDAS}

Mientras tanto, la obra de las Naciones Unidas ha seguido adelante. La Organización ha hecho terminar guerras en distintas partes del mundo y ha intensificado la.colaboración irternacional en casi todos los campos de las actividades humanas, en una escala nunca igualada anteriormente.

Las Naciones Unidas han sido llamadas a actuar en la mayor parte de las zonas perturbadas del mundo. La Organización se ha ocupado de los problemas de Berlín, Palestina, Cachemira e Indonesia; hay también una Comisión Especial de las Naciones Unidas que actúa en los Balcanes, y otra en Corea. Como las grandes potencias no han podido resolver el destino de las colonias italianas, se ha apelado a las Naciones Unidas para que decidan sobre el caso.

Las Naciones Unidas han proclamado la primera Declaración Universal de Derechos del. Hombre; la histórica Convención para la prevención y el castigo del delito de genocidio ha sido aprobada unánimemente y se ha invitado a los estados miembros a que la firmen y ratifiquen.

Por los medios previstos en la Carta, y en particular por el régimen internacional de administración fiduciaria, las Naciones Unidas ayudan a los pueblos dependientes a avanzar en su camino hacia la autonomía o la independencia. 
Las decisiones y las opiriones consultivas de la Corte Internacional de Justicia y la labor de la Comisión de Derecho Internacional, así como las recomendaciones y convenciones aprobadas por la Asamblea General, sientan las bases de un derecho mundial.

A pesar de todas las diferencias políticas e ideológicas, las Nacior.es Unidas elaboran programas de desarrollo económico y social que tienden a elevar el nivel de vida de los países menos desarrollados y a.acrecentar el sentido de responsabilidad de la comunidad de las naciones por el bienestar de cada uno de sus miembros.

Por los métodos de cooperación económica regional, las Naciones Unidas prestan ayuda efectiva a los países de Europa, de América Latina, de Asia y del Lejano Oriente para la reconstrucción y el desarróllo de sus economías.

Por la organización y ejecución de vastos programas en materia económica y social, por el otorgamiento de becas, el envío de misiones y de consejeros y la organización de seminarios regionales, las Naciones Unidas ya han empezado a prestar ayuda efectiva para poner los conocimientos de las naciones más adelantadas a disposición de las que lo están menos.

Uno de los acontecimientos más alentadores del año ha sido el impulso dado, por iniciativa de los Estados Unidos de América, a un programa ampliado de asistencia técnica para el desarrollo económico de las regiones del mundo insuficientemente desarrolladas, que ha de ser puesto en práctica, siempre que sea posible, por conducto de las Naciones Unidas y de los organismos especializados. A solicitud del Consejo Económico y Social, preparé un informe sobre este punto, en consulta con los jefes de los citados organismos. Espero que el programa ampliado de asistencia que so propone en ese informe ha de ser cumplido, y creo que el apoyo activo de los miembros de las Naciones Unidas a este programa facilitará en forma cansiderable la transición pacífica hacia un orden mundial más próspero.

Cuando los acontecimientos internacionales han provocado graves perurias, las Naciones Unidas se han esforzado en remediarlas rápidamente, for todos los medios de que disponen, socorriendo especialmente a las personas que no pueden contar con la asistencia y el apoyo de una comunidad nacional, tales como los refugiados y las personas desalojadas eni Europa, Palestina y el Lejano Oriente.

Aunque la obra económica y social de las Niaciones Unidas y de los organismos especializados está en su iniciación, millones de personas en el mundo entero están ahora en mejores condiciones que antes, gracias a la labor realizada por los organismos especializados y las comisiones de las Naciones Unidas, en cuestiones de trabajo, sanidad, nutrición, agricultura, educación, refugiados, protección a la infancia, desarrollo y estabilidad económicos, finanzas y comercio, fiscalización de estupefacientes y muchas otras cuestiones conexas.

Sin las Naciones Unidas, la mayoría de estas actividades no se habrían emprendido, ni logrado los beneficios consiguientes. Pocas de estas actividades han encontrado obstáculos graves, y aun menos se han visto paralizadas, a causa del conflicto entre grandes potencias. Por último, las tensiones políticas $\mathbf{y}$ otros efectos lamentables del conflicto han sido atenuados considerablemente por muchas de estas actividades de las Naciones Unidas. 


\section{EL PROBLEMA DE LA UNANIMIDAD DE LAS GRANDES POTENCIAS}

Por el contrario, en lo concerniente a la aplicación del artículo 43 de la Carta, al coritrol de la energía atómica y de otras armas de destrucción en masa, y a la reglamentación y reducción de otros armamentos, las diferencias entre las grandes potencias impidieron todo progreso apreciable. Precisamente en esto es esencial la unanimidad de las grandes potencias y esta unanimidad sería igualmente necesaria si no existiera la facultad del veto en el Consejo de Seguridad. Tales problemas sólo pueden resolverse por un acuerdo entre todas ellas.

Creo que debemos reconocer ahora que, en el pasado, se ha insistido demasiado, y sin razón, sobre las funciones coactivas del Consejo de Seguridad. Estas funciones son importantes, y es lamentable que el artículo 43, según el cual se han de poner fuerzas armadas a disposición del Consejo de Seguridad, aún no se haya cumplido. Mientras no se cumpla esta obligación, que impone la Carta, la autoridad de las Naciones Unidas no pcdrá ser tan grande como debiera.

Hay que reconocer, no obstante, que la experiencia de los últimos años ha demostrado que los instrumentos esenciales para la conservación de la paz son los procedimientos de arreglo pacífico. Según el texto de la Carta, éstos son "la negociación, la investigación, la mediación, la conciliación, el arbitraje, el arreglo judicial, el recurso a organismos o acuerdos regionales". Las Naciones Unidas han obtenido ya éxitos considerables en el empleo de estos instrumentos.

En cuanto concierne a las grandes potencias, estos instrumentos de arreglo pacífico son los únicos que puede emplear en la práctica el Consejo de Seguridad. La regla de la unanimidad se aplica a todas las decisiones relativas a medidas coercitivas y, aún en el cáso de que no existiera esta regla la situación no cambiaría esencialmente. Las citadas medidas contra una gran potencia no serían meramente de policía, sino que traerían la guerra; en realidad, una nueva guerra mundial.

Un nuevo conflicto de esta naturaleza, llevada a cabo con los medios actualmente disponibles, sólo podría conducir al mundo a su destrucción. Ningún sistema o ideología prevalecerían después, porque no habría vencedores ni vencidos. Se llegaría al caos total.

Impedir que estalle una nueva guerra mundial es la principal razón de existir de las Naciones Unidas.

Conviene que los pueblos del mundo hagan frente a estos hechos y comprendan que es imposible obtener garantías duraderas contra la guerra mediante un arreglo que .excluya a cualquiera de las grandes potencias. Aunque los acuerdos regionales de seguridad puedan a veces establecer el equilibrio de poderes en el mundo, la seguridad colectiva sólo podrá obtenerse por la aplicación de medios que permitan a las grandes potencias vivir en paz unas con otras bajo la égida de la Carta, aún cuando sea largo el tiempo que se tarde en lograrlo.

Los acontecimientos de los últimos cuatro años y las divergencias entre las grandes potencias hacen más necesaria, y no menos, la existencia de las Naciones Unidas. El único modo de resolver pacíficamente esas di- 
vergencias corsiste en hacer que las Naciones Unidas actúen eficazmente er: el ámbito de las atribuciones que les confiere la Carta.

Otros acontecimientos de nuestro tiempo aumentan la importancia de las Naciones Unidas año tras año. A dos hechos deseo referirme en particular, a saber: al cambio de muchos pueblos de Asia y de Africa, de una situación de dependencia a una de igualdad, y a la fuerza creciente del movimiento en favor de la generalización del respeto a los derechos del hombre en el mundo entero.

Si la importancia de estos hechos se avaluara exactamente, creo que el mundo podría hacer frente con más serenidad al problema actual de las divergercias entre las potencias "orientales" y las "occidentales", pues dichas divergencias serían mejor apreciadas en su verdadera perspectiva. Estimo que la evolución frogresiva de los pueblos dependientes y el movimiento en favor de los derechos del hombre, a la larga, tendrán más significado y provocarán consecuencias históricas en la segunda mitad del siglo $\mathrm{XX}$, que la lucha ideológica actual.

\section{EVOLUCION DE LOS PUEBLOS DEPENDIENTES}

La India, el Pakistán, Birmania y Ceilán, que eran anteriormente partes dependientes del Imperio Británico, han logrado la independencia, por transición pacífica, después de la fundación de las Naciones Unidas, gracias principalmente al alto grado de sentido político demostrado por los gobiernos de esos países y por el Reino Unido. En mi memoria del año pasado puse de manifiesto el sorprendente aumento del número de naciones independientes asiáticas y africanas representadas en la Organización, a partir de la Conferencia de San Francisco. Israel es actualmetne el quincuagésimo noveno miembro de la Organización. Las solicitudes de admisión de otros cuatro países asiáticos -la República Popular de Mongolia, Ceilán, Nepal y Corea- están pendientes de resolución. Puede esperarse que Indonesia presente una solicitud cuando se hayan resuelto las dificultades actuales. En la Asamblea General, el debate sobre las colonias italianas ha puesto de manifiesto que la mayoría de los miembros tiene la intención de recibir a Libia, como miembro independiente, dentro de pocos años; y a la Somalia, un poco más tarde. Podemos estar seguros de que, con el tiempo, otros países de Africa y de Asia ingresarán en la Organización. Así debería ser, pues más de la mitad de la población del mundo vive en esos dos continentes.

La era de dependercia o de subordinación de los pueblos asiáticos sc aproxima rápidamente a su fin. Herederos de algunas de las más grandes civilizacionés de la humanidad, esos pueblos están edificardo su propio nuevo mundo sobre los cimientos del antiguo. Su influencia en los asuntos de las Naciones Unidas aumenta sin cesar. Con el tiempo, y con el necesario desarrollo de sus recursos económicos los países de Asia alcanzarán una influencia que corresponderá mejor a la importancia de sus poblaciones. Es ilusoria la idea de que esta vasta diversidad de pueblos antiguos, que pugnan por una nueva vida y por la independencia, pueda convertirse en mero reflejo de formas de sociedad que existen en otras partes del mundo.

En Africa, la evolución es más lenta. Allá, el régimen internacional de administración fiduciaria puede desempeñar un papel especialmente 
importante, y los efectos benéficos de la inspección de las Naciones Unidas en la administración de los territorios sometidos a tal régimen se advertirán en: la región mucho más vasta de los territorios no autónomos. Los efectos del sistema de administración fiduciaria darán, ciertamente, un impulso al progreso económico, social y cultural de los pueblos de estos territorios, y los conducirán, en definitiva, hacia la autonomía o la independencia.

\section{EL MOVIMIENTO}

\section{EN FAVOR DE LOS DERECHOS DEL HOMBRE}

En el año pasado se ha producido uro de los acontecimientos históricos más significativos en la lucha para generalizar el respeto a los derechos del hombre en el mundo: la proclamación, por la Asamblea General, de la Declaración Universal de Derechos del Hombre. Es ésta la primera vez que se ha redactado una declaración tal para el mundo entero.

En verdad, esta Declaración no tiene fuerza de ley, pero tampoco la han tenido algunos de los documentos que han ejercido más influencia en la historia. La Declaración de Independencia de los Estados Unidos y la Declaración francesa de Derechos del Hombre no tenían fuerza de ley cuando fueron proclamadas.

Sin embargo, la Declaración Universal de Derechos del Hombre tiene trás de si la fuerza de la opinión pública, y en ésta radica todo derecho, sea nacional o internacional. La Declaración establece normas que los gobiernos deben acatar. La influencia benéfica de esta Declaración se ejercerá donde quiera que haya personas privadas de su libertad a causa de sus creencias, o que sean víctimas de una discriminación por el color de su piel o por cualquier otro motivo, o que no puedan lograr condiciones de vida justas, educar a sus hijos y gozar de garantías en caso de enfermedad, accidente o vejez.

La Declaración es, además, sólo el primer paso, aunque el más importante dado por las Naciones Unidas para extender a todo el mundo el mayor respeto a los derechos del hombre.

El papel esencial desempeñado por las Naciones Unidas en estos grandes acontecimientos es más patente cada día.

Por otra parte, las Naciores Unidas favorecen la evolución hacia una mayor igualdad de posibilidades entre los pueblos de Asia y Africa y los del mundo occidental, y entre los paises más y menos desarrollados.

Asimismo, las Naciones Unidas impulsan vigorosamente la causa de los derechos del hombre para todos los pueblos, dondequiera que se hallen. Por otra parte, las Naciones Unidas hacen todo lo posible para que estos cambios fundamentales se efectúen con el mínimo de violencia posible. Las Naciones Unidas, cuando intervienen, tratan de favorecer una evolución pacífica $y$ toda su influencia se ejerce para moderar la intensidad de los conflictos y conciliar, hasta donde sea posible, los puntos de vista opuestos. 


\section{LA ASAMBLEA GENERAL COMO UN PODEROSO FACTOR}

En la evolución de las Naciones Unidas, en sus años de formación, se produjo un hecho que considero como una razón importante de su fuerza creciente. Este hecho es la transformación de la Asamblea General en una de las fuerzas pacificadoras más poderosas que el mundo haya conocido.

Existen tres causas principales que explican este hecho. En primer término, todas las naciones están en ella igualmente representadas; muchas de las naciones pequeñas se han expresado desde el principio con gran independencia, y la Asamblea General ha tendido a ejercer una influencia corciliadora y moderadora en los conflictos entre las grandes potencias.

En segundo término, la atención del mundo se ha concentrado en la Asamblea General, más que en ningún otro organismo internacional: sus reuniones periódicas han llegado a ser el más importante campo de experimentación de la política de los estados miembros. Aquí, como en ninguna otra parte, estas políticas están sometidas a un examen severo y al juicio de la opinión pública mundial. Se ha demostrado ya que, cuando una política nacional no sale triunfante de esta prueba de justicia y de lealtad, habrá de ser modificada, si no inmediatamente, por lo menos en el futuro.

En tercer término, la Asamblea General ha demostrado que puede ejercer las atribuciones que le confiere la Carta, para la conciliación y el arreglo pacífico de las controversias, como un complemento poderoso de la labor del Consejo de Seguridad para la prevención de la guerra.

No estoy de acuerdo con las críticas que se formulan contra la Asamblea General, considerándola como un club de debates sin poder alzuno o como una tribuna de propaganda. La mayor parte de las recomendaciones de la Asamblea contribuyen a cimentar un derecho mundial y a impedir la guerra. Cuando un gobierno utiliza la tribuna de la Asamblea para fines de propaganda, tal gobierno se somete al juicio de la humanidad. No obstante, convengo en que los procedimientos de la Asamblea Cieneral podrían ser ferfeccionados, y en que sería posible ganar mucho tiempo, principalmente si los miembros que participan en los debates limitaran sus intervenciones.

Podemos estar convencidos de que las garantías que obtenga el mundo contra la guerra estarán en proporción con el apoyo que den los gobiernos miembros a las Naciones Unidas. El peligro de guerra aumentará en la medida en que estos gobiernos releguen a las Naciones Unidas a ur papel secundario: y las garantías contra la guerra se reforzarán en la medida en que aquéllos hagan de las Naciones Unidas la piedra angular de su polítíca exterior y apoyen por la palabra y por los actos la supremacía de la Carta y la universalidad de la Organización.

\section{CONSOLIDACION DE LAS NACIONES UNIDAS}

Los gobiernos miembros pueden disminuir el peligro de la guerra actuando de modo que las Naciones Unidas se fortalezcan y que sus servicios sean utilizados en diversas formas en los meses venideros. A este 
respecto, tengo que sugerir varias proposiciones que presento a la consideración de la Asamblea.

\section{RELACIONES ENTRE LAS GRANDES POTENCIAS}

Las Naciones Unidas no están en posibilidad de imponer un arreglo del conflicto actual entre las grandes potencias, pero han demostrado que pueden contribuir a suavizar tal conflicto, a atenuarlo y a contenerlo en límites pacíficos. Es impresciridible que las grandes potencias se mantengan en contacto, discutan sus problemas y entablen entre sí negociaciones sinceras. Rechazar las consultas for inútiles equivale a negar el fundamerto mismo de las Naciones Unidas. Ahora cuando las potencias occidentales y la Unión de Repúblicas Socialistas Soviéticas han reanudado sus debates en el Consejo de Ministros de Relaciones Exteriores y manifestado su intención de continuarlos durante el periodo de sesiones de la Asamblea General, espero que no habrán de abandonar sus esfuerzos para resolver sus diferencias.

Tarde o temprano debe haber soluciones de paz para Alemania, Austria y el Japón; debe haber ur acuerdo para poner a disposición del Consejo de Seguridad fuerzas armadas, como para el control de la energía atómica y de otros medios de destrucción en masa, y asimismo sobre los armamentos de tipo corriente. Es necesario continuar esforzándose por adelantar en estas materias aunque las perspectivas sean desalentadoras en determinado momento o se requieran muchos años para llevar a cabo la tarea. Confío en que la práctica de realizar consultas entre las grandes potencias constituirá la regla y no la excepción de los asuntos planteados ante las Naciones Unidas, especialmente ante el Consejo de Seguridad, del misr.o modo que en los asuntos que están fuera de la competencia de la Organización. Los buenos oficios del Secretario General están siempre a disposición de los miembros para facilitar estas consultas.

\section{SERVICIO MOVIL DE LAS NACIONES UNIDAS}

Con el fin de hacer más eficaz la labor de mediación y conciliación. durante el último período de sesiones de la Asamblea General propuse el establecimiento de una Guardia de las Naciones Unidas, que no tendría obligaciones militares, pero asistiría a las misiones de las Naciones Unidas que llevan a cabo sus tareas pacíficas en las zonas de perturbación del mundo. En vista de las indicaciones hechas por los gobiernos miembros, he modificado aquella proposición: he propuesto a la Comisión Especial creada por la Asamblea General, que se establezca un Servicio Móvil de las Naciones Unidas, compuesto de 300 hombres de uniforme. Estos hombres, seleccionados según el principio de la distribución geográfica, serían proporcionados por los estados miembros o tomados de los servicios públicos de dichos estados, por un período de uno a tres años; prestarian servicio de protección y desempeñarían funciones técnicas en la sede de la Organización y en las misiones de ésta. También he sugerido la creación de un cuadro de reserva de 2 mil hombres, que podrían ocuparse de la observación de las treguas y de las obligaciones impuestas por los plebiscitos, y que los órganos competentes de las Naciones Unidas Ilamarian al 
servicio cuando surgiera la necesidad. Ninguno de estos hombres del Servicio Móvil llevaría armas, excepto las de cinto para propia protección en caso necesario. Según el plar revisado, el costo para la Organización se mantendría en un mínimo, y las ventajas de la proposición original serían conservadas. La Secretaría, que ha mejorado constantemente su eficiencia, dispordría así de los medios necesarios para hacer frente a sus crecientes responsabilidades, y el poder y el prestigio de las Naciones Unidas en el arreglo pacífico de las controversias se verían reforzados.

\section{LAS COLONIAS ITALIANAS}

La Asamblea General en su tercer período ordinario de sesiones, no llegó a un acuerdo sobre el destino que ha de darse a las antiguas colonias italianas. La cuestión se planteará nuevamente este otoño en el cuarto período de sesiones.

El debate sobre esta cuestión, en la segunda parte del tercer período de sésiones, es significativo en varios aspectos. En él se reflejó la influencia creciente de las naciones de Asia y de Africa. Estas naciones desempeñaror un papel decisivo para impedir que se adoptara una propuesta transaccional que, en su opinión, no satisfaría a la mayoría de los habitantes de los territorios interesados. El debate también puso de manifiesto el apoyo considerable que logra la idea de una administración fiduciaria directa de las Naciones Unidas, según la propuesta presentada originalmente por el Gobierno de los Estados Unidos en la primera reunión del Consejo de Ministros de Relaciones Exteriores efectuada en Londres.

Reconozco. las dificultades de orden político y práctico que entraña una administración fiduciaria directa de las Naciones Unidas sobre esos territorios, durante el periodo relativamente breve que preceda a su independencia. Creo, sin embargo, que el buen sentido político de los gobiernos podría conducir a una solución del problema, y que las dificultades de orden político podrían obviarse si las de orden práctico fueran resueltas. La mejor solución, en mi concepto, consiste en una administración fiduciaria directa de las Naciones Unidas, con un administrador responsable tan sólo ante el Consejo de Administración Fiduciaria. Naturalmente, la decisión corresponde a los gobiernos miembros, pero estoy seguro de que un paso tan audaz ayudaría a los pueblos de los territorios interesados a seguir el camino de paz que lleva a la autonomía o a la independencia, y fortalecería la confianza de todos los pueblos dependientes en las Naciones Unidas y en los gobiernos miembros que hubieran hecho posible tal solución.

\section{LA PAZ Y EL DESARROLLO ECONOMICO DEL CERCANO ORIENTE}

El Cercano Oriente ofrece una oporturidad sin igual a las Naciones Unidas para conjugar la actividad política cor la económica, a fin de obtener una paz duradera.

Un armisticio se ha concertado en Palestina, y se están negociando las condiciones de un arreglo pacífico. Novecientos mil árabes reciben asistencia de las Naciones Unidas; muchos de ellos deben ser repatriados $\sigma^{*}$ 
reinstalados. El nuevo estado de Israel tiene aún que alcanzar la viabilidad económica. Tanto Israel como los estados árabes necesitan realizar un esfuerzo poderoso y coordinado de desarrollo económico, para elevar sus condiciones de vida.

Estimo que esta región debe obtener precedencia en el programa propuesto por las Naciones Unidas para la asistencia técnica a los países poco desarrollados. Esto permitiría elaborar amplios planes para el desarrollo económico en gran escala de toda la región; estos planes podrian incluir proyectos de desarrollo de las cuencas fluviales, similares al proyecto del valle del Tennessee en los Estados Unidos, para los valles del Tigris, del Eufrates y del Jordán, y para el desarrollo ulterior del valle del Nilo. El primer paso es la asistencia técnica: el segundo, las inversiones financieras. Si se acometen ambos por conducto de las Naciones Unidas, los gravámenes, lo mismo que los beneficios, pueden distribuirse equitativamente entre los países farticipantes.

El desarrollo del Cercano Oriente no deberá impedir, por supuesto, ni demorar, los programas similares de asistencia técnica y desarrollo económico en otras partes de Asia o de Africa, ni en la América Latina.

\section{ACCION PARA RESOLVER LAS DIFICULTADES ECONOMICAS}

Aunque la situación económica mundial haya mejorado considerablemente en muchos aspectos, las condiciones básicas de estabilidad económica y desarrollo ordenado no se han establecido aún.

Particular importancia tiene el desequilibrio continuo y persistente del comercio y de los pagos internacionales. Escasos adelantos se han realizado hacia la solución de este grave problema. Las consecuencias de ello han sido especialmente perjudiciales para los países más débiles y menos favorecidos.

Por otra parte, si se permite que continúe la reciente disminución de actividad económica en algunos países, esto causará en ellos una desocupación en gran escala, y al mismo tiempo agravará los actuales desajustes económicos en el mundo entero. Otra crisis económica de alcance mundial sería una tragedia, y esta tragedia podría prevenirse si los gobiernos del mundo encontraran una base común para una acción concertada. La solución para estos problemas, ènormemente complicados, no podrán obtenerla los países aislados, actuando por si solos, ni tampoco un grupo limitado de naciones.

Afortunadamente, los gobiernos miembros tienen a su disposicion, en las Naciones Unidas y en los organismos especializados, el mecanismo interracional' que les permite desarrollar la necesaria acción concertada. Urge que utilicen este mecanismo para hacer frente a estos apremiantes problemas económicos.

Las solicitudes de catorce países para ingresar en las Naciones Unidas, están pendientes de resolución. Estos países son, por orden cronológico de sus solicitudes: Albania, la República Popular de Mongolia, Jordania, Portugal, Irlanda, Hungría, Italia, Austria, Rumanía, Bulgaria, Finlandia, Ceilán, Corea y Nepal. La mayoría están en espera de su admisión hace más de dos años. 


\section{UNIVERSALIDAD DE LA ORGANIZACION}

Los gobiernos miembros conocen bien mi opinión sobre la conveniencia de favorecer, en la forma más rápida posible, la universalidad de las Naciones Unidas. La he expresado en varias ocasiones durante los últimos tres años. No ignoro las objeciones que se han formulado contra la admisión de aquellos estados, pero creo que tales objeciones podrían ser examinadas mejor si los solicitantes pertenecieran a la Organización, en: vez de estar fuera de ella. Puede considerarse que los solicitantes, de todos modos, reúnen las condiciones necesarias para el ingreso. Dígase lo que se quiera sobre el gobierno de los países interesados, sus pueblos y el mur.do entero se beneficiarían indudablemente, si todos los solicitantes fueran admitidos en la Organización del próximo período de seșiones de la Asamblea General.

Recomiendo a los gobiernos miembros y a los pueblos del mundo el estudio del informe completo sobre la actividad de las Naciones Unidos erı los doce meses que terminaron el 30 de junio de 1949. En ellos se da cuenta de los éxitos obtenidos en la prevención de la guerra y en el establecimiento progresivo de los cimientos de ur. mundo más pacífico y más próspero. 